\title{
Effect of magnetic field on the propagation of quasi-transverse waves in a nonhomogeneous conducting medium under the theory of nonlinear elasticity
}

\author{
D P ACHARYA ${ }^{1}$ and ASIT KUMAR MONDAL ${ }^{2}$ \\ ${ }^{1}$ 109/3, Kailash Roy Chowdhury Road, Barrackpore, Kolkata 700 120, India \\ ${ }^{2}$ Kidderpore College, 2 \& 3 Pitamber Sircar Lane, Kolkata 700 023, India \\ e-mail: acharyadp_05@ rediffmail.com
}

MS received 29 June 2005; revised 2 January 2006

\begin{abstract}
The object of the present paper is to investigate the propagation of quasi-transverse waves in a nonlinear perfectly conducting nonhomogeneous elastic medium in the presence of a uniform magnetic field transverse to the direction of wave propagation. Different types of figures have been drawn to exhibit the distortion of waves due to the presence of magnetic field and the nonhomogeneous nature of the medium. Formation of shocks has also been numerically discussed.
\end{abstract}

Keywords. Electromagnetic field; quasi-transverse waves; nonlinear wave motion; nonhomogeneity; multiple scale technique; distortion of waves; formation of shocks.

\section{Introduction}

In the classical theory of elasticity, it is assumed that elastic bodies undergo deformation due to external forces within the frame work of infinitesimal strain theory, where constitutive equations are taken to be linear. Utilizing the above linear theory, the effects of magnetic field, nonhomogeneity, surface stress, voids etc., on the propagation of waves have also been investigated by many authors (Yu et al 1966; Paria 1967; Chandrasekharaiah 1987a,b; Pal \& Acharya 1998). Such a linear approximation may not reflect the actual effects in many cases, e.g. it cannot explain the Kelvin effect and the Poynting effect (Truesdell \& Noll 1965). Nonlinear formulations, therefore, have their importance in the formations of finite amplitude waves, magneto-elastic waves etc. They also play a vital role in the study of the interaction of the nonhomogeneity of the magneto-elastic material medium with the nature of nonlinear waves. Moreover, for proper understanding of the phenomena of distortion and the formation of shocks, we have to consider the propagation of waves in a nonlinear elastic medium.

Interplay of electromagnetic field with the motions of a deformable solid has its importance owing to its theoretical and practical relevance in various branches of science and technology. A survey of linear and nonlinear wave motion in a perfect magneto-elastic medium has been made by Bazer (1984). Nayfeh (1975) considered one-dimensional problems related to the propagation of longitudinal and transverse waves in a nonlinear medium. 
Semra \& Mevlut (2004) investigated Rayleigh-like surface waves on a nonlinear layered elastic half-space. Using perturbation and multiple scale techniques, Lardner (1983) and Bazer (1984) investigated the growth of amplitude and shock formation in the said wave propagation. Maugin (1981) discussed a few problems related to the propagation of waves in magnetizable elastic solids. Hefni et al (1995) considered surface waves in a nonlinear magneto-elastic conductor of finite electric conductivity. Recently, Chakraborty (1998) investigated the distortions of longitudinal waves and formation of shocks due to elastic nonlinearity of the medium in presence of a magnetic field. Eringen \& Suhubi (1974) pointed out that purely plane longitudinal waves exist in a nonlinear elastic medium. Lardner (1985) has discussed the existence of an antiplane shear waves where the lowest order effect of nonlinearity includes a longitudinal displacement of the second order. In other words, for a nonlinear elastic medium, there exists a type of wave which travels in the $x_{1}$-direction with predominant displacement in the transverse direction $x_{3}$ and higher order displacement in the $x_{1}$-direction. Such waves are termed quasi-transverse waves. Chakraborty (1994), Ang \& Clements (1987) and Chattopadhyay et al (1984) pointed out that nonhomogeneity of the medium modulates the propagation of waves to a considerable extent in many cases. Investigation of the present problem continues with the previous sequence of work and is concerned with the more general case of the solution of the problem of quasi-transverse wave propagation, taking into account the nonhomogeneity of the material medium and the presence of the magnetic field together with the variation of magnetic permeability.

The nonhomogeneity of the elastic material is such that it grows or decays slowly, depending on the space variable according to which it varies. In the present problem we have characterised the nonhomogeneity by supposing that the elastic compliances depend on the value at the point under consideration and its rate of growth or decay is proportional to its value at the point and as such we take, for example,

$$
\mathrm{d} \lambda / \mathrm{d} x=v_{1} \lambda
$$

where $v_{1}$ is constant, being positive for growth and negative for decay. In other words, $v_{1}$ may be considered as a growth parameter where it is positive and decay parameter where it is negative, and $\lambda$ is an elastic modulus. Clearly, integrating (1), we get

$$
\lambda=\lambda_{0} e^{v_{1} x}
$$

where $\lambda_{0}$ is the value of $\lambda$ when $v_{1}=0$ (homogeneous medium).

In the present problem, we shall at first consider a nonhomogeneous material medium with exponentially diminishing density and elastic parameters $\left(v_{1}=-v, v>0\right)$. Hence the density $(\rho)$, Lamé moduli $(\lambda, \mu)$ and the third order elastic parameters may be presented in the following forms:

$$
\rho=\rho_{0} e^{-v x}, \lambda=\lambda_{0} e^{-v x}, \mu=\mu_{0} e^{-v x}, \alpha=\alpha_{0} e^{-v x}, \beta=\beta_{0} e^{-v x}, \gamma=\gamma_{0} e^{-v x},
$$

where the constants $\rho_{0}, \lambda_{0}, \mu_{0}, \alpha_{0}, \beta_{0}, \gamma_{0}$ correspond to those for a homogeneous medium $(v=0)$. The case of a nonhomogeneous medium with exponentially increasing parameters $(v<0)$ will be discussed in due course.

It is believed that the problem, as considered in its present form, has not been discussed so far. 


\section{Basic equations and formulations of the problem}

Maxwell's equations (neglecting the displacement current) of electromagnetic field are given by

$$
\begin{aligned}
& \operatorname{div} \mathbf{B}=0, \\
& \operatorname{div} \mathbf{D}=0, \\
& \operatorname{curl} \mathbf{H}=\mathbf{J}, \\
& \operatorname{curl} \mathbf{E}=-\mathbf{B}_{t}
\end{aligned}
$$

where $\mathbf{B}=$ magnetic induction vector, $\mathbf{D}=$ electric displacement vector, $\mathbf{H}=$ magnetic field vector, $\mathbf{J}=$ current density vector, $\mathbf{E}=$ electric field vector and suffix $\mathbf{t}$ represents the differentiation with respect to time. Moreover,

$$
\begin{aligned}
& \mathbf{B}=\mu_{e} \mathbf{H}, \\
& \mathbf{D}=\varepsilon_{1} \mathbf{E},
\end{aligned}
$$

where $\mu_{e}$ is the magnetic permeability and $\varepsilon_{1}$ is the electric permittivity. Here, we assume that $\mu_{e}$ is not a constant but varies exponentially as follows:

$$
\mu_{e}=\mu_{e_{0}} e^{-v x},
$$

where $\mu_{e_{0}}$ is the value of $\mu_{e}$ when the parameter $v=0$.

Again, Ohm's law states that

$$
\mathbf{J}=\boldsymbol{\sigma}\left(\mathbf{E}+\mathbf{u}_{t} \times \mathbf{B}\right), \boldsymbol{\sigma} \text { being the electrical conductivity. }
$$

For the propagation of nonlinear quasi-transverse waves travelling in the $x_{1}$-direction in a perfectly conducting medium in presence of a uniform magnetic field $\mathbf{H}^{0}$ transverse to the direction of the propagation of waves, the stress equation of motion referred to rectangular Cartesian coordinates $\left(x_{1}, x_{2}, x_{3}\right)$ in a conducting medium may be written as

$$
\begin{gathered}
L_{i j, j}+(\mathbf{J} \times \mathbf{B})_{i}=\rho u_{i, t t} \\
\text { where } L_{i j}=\text { Piola-Kirchhoff stress tensor }=\partial W / \partial u_{i, j},
\end{gathered}
$$

where $W$ is the strain energy density function of the material medium per unit volume, $(, j)$ denotes $\left(\partial / \partial x_{j}\right)$, repeated indices indicate summation with respect to that index, $\mathbf{J} \times \mathbf{B}$ is the Lorentz force and $u_{i}=\left(u_{1}, u_{2}, u_{3}\right)$ denotes the material displacement.

Green's strain tensor is given by (Bland 1969, Chakraborty 1998)

$$
e_{i j}=\frac{1}{2}\left[u_{i, j}+u_{j, i}+u_{k, i} u_{k, j}\right], i, j, k=1,2,3 .
$$

We may write the strain energy density function $W$ in terms of strain invariants as follows (Bland 1969; Chakraborty 1998):

$$
W=\frac{1}{2} \lambda I_{1}^{2}+\mu I_{2}+\alpha I_{1}^{3}+\beta I_{1} I_{2}+\gamma I_{3},
$$


where approximation, correct up to the third order in displacement gradient, has been taken into account and $\lambda, \mu$ are Lamé's elastic constants whereas $\alpha, \beta, \gamma$ are third-order elastic constants. Moreover,

$$
I_{1}=e_{i i}, I_{2}=e_{i j} e_{j i}, I_{3}=e_{i j} e_{j k} e_{k i},
$$

are strain invariants.

Following Lardner (1985) and Chakraborty (1994) for a quasi-transverse wave travelling in the $x_{1}$-direction with predominant displacement in the transverse direction $x_{3}$, and higher order displacement in the $x_{1}$-direction we take displacement components as

$$
u_{1}=\varepsilon^{2} u(x, t), \quad u_{2}=0, \quad u_{3}=\varepsilon w(x, t),
$$

where $\varepsilon$ is a small scaling parameter, $u(x, t)$ and $w(x, t)$ are quantities of $0(1)$ in which $x_{1}$ has been changed to $x$. The choice of $\varepsilon$ has been made in such a manner that the lowest order effect of nonlinearities includes a longitudinal displacement of second order (Chakraborty 1994, 1998).

To suit the actual situation of the problem, the nonzero components of strain are given by

$$
e_{11}=e_{x x}=\varepsilon^{2}\left[u_{x}+\frac{1}{2} w_{x}^{2}\right]+0\left(\varepsilon^{4}\right), \quad e_{13}=e_{x z}=\frac{1}{2} \varepsilon w_{x}
$$

where $u_{x}=\partial u / \partial x, w_{x}=\partial w / \partial x$.

The relevant stress components which are due to Piola-Kirchhoff may be written as

$$
\begin{aligned}
& L_{11}=\varepsilon^{2} \mu\left[A u_{x}+B w_{x}^{2}\right]+0\left(\varepsilon^{4}\right) \\
& L_{13}=\varepsilon \mu w_{x}+\varepsilon^{3} \mu\left[\left\{\frac{\mu+\beta+\frac{3}{2} \gamma}{\mu}\right\} u_{x} w_{x}+\left\{\frac{\beta+\frac{3}{2} \gamma}{2 \mu}\right\} w_{x}^{3}\right]+0\left(\varepsilon^{5}\right) \\
& L_{22}=\varepsilon^{2} \mu\left[(A-2) u_{x}+\frac{\lambda+\beta}{2 \mu} w_{x}^{2}\right]+0\left(\varepsilon^{4}\right) \\
& L_{33}=\varepsilon^{2} \mu\left[(A-2) u_{x}+B w_{x}^{2}\right]+0\left(\varepsilon^{4}\right) \\
& L_{31}=\varepsilon \mu w_{x}+\varepsilon^{3} \mu\left[C u_{x} w_{x}+D w_{x}^{3}\right]+0\left(\varepsilon^{5}\right) \\
& L_{12}=L_{21}=L_{23}=L_{32}=0
\end{aligned}
$$

where

$$
\begin{aligned}
A & =(\lambda+2 \mu) / \mu, & B & =\left(\lambda+\beta+2 \mu+\frac{3}{2} \gamma\right) / 2 \mu, \\
C & =\left(\lambda+2 \mu+\beta+\frac{3}{2} \gamma\right) / \mu, & D & =(\lambda+\beta+2 \mu+3 \gamma) / 2 \mu .
\end{aligned}
$$

In the present investigation, we shall keep in mind the exponential variation of density, elastic moduli to express the nonhomogeneous character of the material medium.

Thus, for the considered problem the relevant equations of motion may be taken as follows:

$$
\begin{aligned}
& L_{11,1}+(\mathbf{J} \times \mathbf{B})_{1}=\rho \ddot{u}_{1}, \\
& L_{31,1}+(\mathbf{J} \times \mathbf{B})_{3}=\rho \ddot{u}_{3} .
\end{aligned}
$$


Since the initially uniform magnetic field $\mathbf{H}^{0}$ is transverse to the direction of wave propagation, we take

$$
\mathbf{H}^{0}=\left(0,0, H^{0}\right)
$$

The perturbed electromagnetic fields are taken as

$$
\begin{aligned}
\mathbf{E} & =\mathbf{e}, \\
\mathbf{H} & =\mathbf{H}^{0}+\mathbf{h}, \\
\mathbf{J} & =\mathbf{j},
\end{aligned}
$$

where the initial values of $\mathbf{E}$ and $\mathbf{J}$ are null vectors.

Since the medium is a perfectly conducting one, we have

$$
\mathbf{E}+\mathbf{u}_{t} \times \mathbf{B}=0 .
$$

From (7), (8), (28), (29) we obtain

$$
\operatorname{curl} \mathbf{e}=-\mu_{e} \mathbf{h}_{t} .
$$

For the perturbed part of the magnetic field, we take

$$
\mathbf{h}=\left(h_{1}, h_{2}, h_{3}\right) .
$$

From (31), by using (32), we have

$$
\mu_{e} \mathbf{h}_{t}=\operatorname{curl}\left(\mathbf{u}_{t} \times \mu_{e} \mathbf{H}\right) .
$$

Now, using the above equations, the components of $\mathbf{h}_{t}$ may be written as follows:

$$
\begin{aligned}
h_{1_{t}}= & 0, \\
h_{2_{t}}= & v h_{2} u_{1_{t}}-h_{2_{x}} u_{1_{t}}-h_{2} u_{1_{t x}}, \\
h_{3_{t}}= & -v h_{1} u_{3_{t}}+h_{1_{x}} u_{3_{t}}+h_{1} u_{3_{t x}}+v\left(H^{0}+h_{3}\right) u_{1_{t}}-\left(H^{0}+h_{3}\right)_{x} u_{1_{t}} \\
& -\left(H^{0}+h_{3}\right) u_{1_{t x}},
\end{aligned}
$$

in which $h_{1_{t}}=\left(h_{1}\right)_{t}=\left(\partial h_{1} / \partial t\right)$ etc.

From (35) it is seen that $h_{1}$ is independent of $t$ and hence it is a function of $x$ alone and we take $h_{1}=0$.

Hence $\mathbf{h}=\left(0, h_{2}, h_{3}\right)$, where $h_{2}$ and $h_{3}$ may be taken in the form of perturbation expressions as follows:

$$
\begin{aligned}
& h_{2}(x, t)=\varepsilon h_{20}(x, \xi, t)+\varepsilon^{2} h_{21}(x, \xi, t)+0\left(\varepsilon^{3}\right), \\
& h_{3}(x, t)=\varepsilon^{2} h_{30}(x, \xi, t)+0\left(\varepsilon^{4}\right) .
\end{aligned}
$$

Using (38), (39) in (36) and (37) and equating the coefficients of $\varepsilon$ and $\varepsilon^{2}$ we get,

$$
\begin{aligned}
& h_{20_{t}}=0, \\
& h_{21_{t}}=0, \\
& h_{30_{t}}=v H^{0} u_{t}-H^{0} u_{t x} .
\end{aligned}
$$


Here $h_{20_{t}}=\left(h_{20}\right)_{t}=0$ and $h_{21_{t}}=\left(h_{21}\right)_{t}=0$ indicate that both are independent of $t$ which implies that $h_{20}=0$ and $h_{21}=0$.

Thus, from (38), $h_{2}(x, t)$ may be approximated to zero.

Therefore, $\mathbf{J} \times \mathbf{B}=\left[-\mu_{e}\left(H^{0}+h_{3}\right) h_{3_{x}}, 0,0\right]$.

The equations of motion (25) and (26) are transformed with the help of (43) to

$$
\begin{aligned}
\left(1 / C_{T}^{2}\right) u_{t t}-A u_{x x}= & 2 B w_{x} w_{x x}-v\left[A u_{x}+B w_{x}^{2}\right] \\
& +\left(a_{v}^{2} / C_{T}^{2}\right) u_{x x}-\left(a_{v}^{2} / C_{T}^{2}\right) v u_{x}+0\left(\varepsilon^{2}\right), \\
\left(1 / C_{T}^{2}\right) w_{t t}-w_{x x}= & \varepsilon^{2}\left[w_{x}\left(C u_{x}+D w_{x}^{2}\right)\right]_{x}-v w_{x} \\
& -v \varepsilon^{2}\left[w_{x}\left(C u_{x}+D w_{x}^{2}\right)\right]+0\left(\varepsilon^{4}\right),
\end{aligned}
$$

where $a_{v}^{2}=\mu_{e_{0}} H^{0^{2}} / \rho_{0}=$ Alfvén wave velocity, and $C_{T}^{2}=\mu_{0} / \rho_{0}=$ shear wave velocity.

\section{Solution of the problem}

In view of the actual context of the problem we take $u(x, t)$ and $w(x, t)$ in the following forms:

$$
\begin{aligned}
u(x, t) & =u_{0}(x, t, \xi)+0\left(\varepsilon^{2}\right), \\
w(x, t) & =w_{0}(x, t, \xi)+\varepsilon^{2} w_{1}(x, t, \xi)+0\left(\varepsilon^{4}\right) .
\end{aligned}
$$

To use the multiple scale technique, following Lardner (1985), we have to introduce the slow distance scale (Nayfeh 1973; Lardner 1985) given by,

$$
\xi=\varepsilon^{2} x .
$$

Moreover, for consistency of the order of magnitude assumption, the nonhomogeneity parameter $v$ may be assumed to be of order $\varepsilon^{2}$. Thus

$$
v=v_{0} \varepsilon^{2} \text {. }
$$

Equations (44) and (45) with the help of (46), (47) and (49) become

$$
\begin{aligned}
& \left(1 / C_{T}^{2}\right) u_{0_{t t}}-\left(A+\left(a_{v}^{2} / C_{T}^{2}\right)\right) u_{0_{x x}}=2 B w_{0_{x}} w_{0_{x x}}+0\left(\varepsilon^{2}\right), \\
& \left(\left(1 / C_{T}^{2}\right) w_{0_{t t}}-w_{0_{x x}}\right)+\varepsilon^{2}\left(\left(1 / C_{T}^{2}\right) w_{1_{t t}}-2 w_{0_{x \xi}}-w_{1_{x x}}\right) \\
& \quad=\varepsilon^{2}\left[w_{0_{x x}}\left(C u_{0_{x}}+D w_{0_{x}}^{2}\right)+\varepsilon^{2}\left[w_{0_{x}}\left(C u_{0_{x x}}+2 D w_{0_{x}} w_{0_{x x}}\right)\right]\right. \\
& \quad-v_{0} \varepsilon^{2} w_{0_{x}}+0\left(\varepsilon^{4}\right) .
\end{aligned}
$$

Hence, the following equations are obtained by equating the terms of different orders of $\varepsilon$

$$
\begin{aligned}
& \left(1 / C_{T}^{2}\right) u_{0_{t t}}-\left(A+\left(a_{v}^{2} / C_{T}^{2}\right)\right) u_{0_{x x}}=2 B w_{0_{x}} w_{0_{x x}}, \\
& \left(1 / C_{T}^{2}\right) w_{0_{t t}}-w_{0_{x x}}=0, \\
& \left(1 / C_{T}^{2}\right) w_{1_{t t}}-w_{1_{x x}}=2 w_{0_{x \xi}}+w_{0_{x x}}\left(C u_{0_{x}}+D w_{0_{x}}^{2}\right) \\
& \quad+w_{0_{x}}\left(C u_{0_{x x}}+2 D w_{0_{x}} w_{0_{x x}}\right)-v_{0} w_{0_{x}} .
\end{aligned}
$$


The solution of (53) in the case of a travelling wave in the positive direction of the $x$-axis may be,

$$
w_{0}(x, t, \xi)=H(\theta, \xi),
$$

where $H$ is a function of $\theta, \xi$ and $\theta=t-x$.

Substituting (55) in (52), we get,

$$
u_{0}(x, t, \xi)=\left[B /\left(1 / C_{T}^{2}\right)\left\{\left(A C_{T}^{2}+a_{v}^{2}\right)-1\right\}\right] \int h^{2}(\theta, \xi) d \theta
$$

where $h=\partial h / \partial \theta$.

In the above we have assumed that $u_{0}$ travels to the right in phase with $w_{0}$.

Putting in the values of $w_{0}$ and $u_{0}$ from (55) and (56), and by using transformation $\theta=$ $t-x, \phi=t+x$ in (54), we obtain

$$
\begin{gathered}
\left(\frac{1}{C_{T}^{2}}-1\right) \frac{\partial^{2} w_{1}}{\partial \theta^{2}}+4 \frac{1}{C_{T}^{2}} \frac{\partial^{2} w_{1}}{\partial \theta \partial \phi}+\left(\frac{1}{C_{T}^{2}}-1\right) \frac{\partial^{2} w_{1}}{\partial \phi^{2}} \\
=-2 H_{\theta \xi}+\frac{2}{3} S \frac{\partial}{\partial \theta} H_{\theta}^{3}+v_{0} H_{\theta}, \\
\text { where } S=\frac{3}{2}\left[D-B C /\left(1 / C_{T}^{2}\right)\left\{\left(A C_{T}^{2}+a_{v}^{2}\right)-1\right\}\right]
\end{gathered}
$$

Following Lardner (1985), units have been so chosen that the shear wave velocity be equal to unity $\left(C_{T}^{2}=\mu_{0} / \rho_{0}=1\right)$.

Therefore, (58) transforms to a simpler form

$$
\begin{aligned}
& 4 \frac{\partial^{2} w_{1}}{\partial \theta \partial \phi}=-2 H_{\theta \xi}+\frac{2}{3} S \frac{\partial}{\partial \theta} H_{\theta}^{3}+v_{0} H_{\theta}, \\
& \text { where } S=\frac{3}{2}\left[D-\frac{B C}{(A-1)+a_{v}^{2}}\right] .
\end{aligned}
$$

Solving (60), we obtain $w_{1}$ in the following form

$$
w_{1}=\frac{1}{2}\left[\frac{1}{3} S H_{0}^{3}-H_{\xi}+\frac{v_{0}}{2} H\right] \phi+\text { complementary function. }
$$

To avoid the secular terms, $H$ must satisfy the following equation,

$$
H_{\xi}-(1 / 3) S H_{\theta}^{3}-\left(v_{0} / 2\right) H=0,
$$

which on differentiation with respect to $\theta$, gives

$$
h_{\xi}-S h^{2} h_{\theta}=\left(v_{0} / 2\right) h
$$

This is a first-order quasi-linear equation and its solution may be obtained as

$$
h(\theta, \xi)=f(\zeta) \exp \left(v_{0} \xi / 2\right) .
$$


In the above, $x=\xi=0$, and $\zeta=\theta=t$ lead to $f(t)=h(t, 0)$, and hence $f(\zeta)$ is the boundary value of $h$ on $x=0$. Moreover, $\zeta$ is connected with $\theta$ as follows

$$
\zeta=\theta+\left(S / v_{0}\right)(f(\zeta))^{2}\left(\exp \left(v_{0} \xi\right)-1\right)
$$

Equations (65) and (66) are similar in form with those obtained by Chakraborty (1994), where the presence of a magnetic field was not considered, the difference lies only in the expression for $S$ in which Alfvén wave velocity, arising due to the presence of magnetic field, is involved.

The above equations help us to study the distortion of a wave form which is not symmetric due to the presence of $(f(\zeta))^{2}$ in (66).

\subsection{Particular cases}

In the absence of the magnetic field, the above results coincide with the corresponding results obtained by Chakraborty (1994).

Again, if the medium be assumed to be homogeneous, making $v_{0} \rightarrow 0$, (65) and (66) transform to

$$
\begin{aligned}
& h(\theta, \xi)=f(\zeta) \\
& \text { and } \zeta=\theta+\frac{3}{2}\left[D-\frac{B C}{(A-1)+a_{v}^{2}}\right] \xi[f(\zeta)]^{2}
\end{aligned}
$$

Equations (67) and (68) represent quasi-transverse wave distortion as the wave progresses in a perfectly conducting elastic homogeneous medium in the presence of the uniform magnetic field $\mathbf{H}^{0}$ transverse to the direction of wave propagation.

Lastly, in the absence of the magnetic field, the corresponding equations for the propagation of one-dimensional quasi-transverse waves in a homogeneous elastic medium may be obtained from (67) and (68) as follows:

$$
\left.\begin{array}{c}
h(\theta, \xi)=f(\zeta) \\
\text { with } \zeta=\theta+S \zeta[f(\zeta)]^{2}
\end{array}\right\}
$$

in which $S=(3 / 2)[D-(B C /(A-1))]$.

This result coincides with the corresponding result of Lardner (1985).

\subsection{Formation of shock waves}

The propagation of the main wave so formed, $w_{0}(x, t, \xi)=H(\theta, \xi)$, depends on the elastic property and the nonhomogeneous character of the material medium. Moreover, it is also seen that the presence of the magnetic field plays a vital role in the above propagation. We now study (65) and (66) for the possibility of formation of shock waves. From the condition of formation of shock wave we have,

$$
\mathrm{d} \theta / \mathrm{d} \zeta=0,
$$

and hence we get

$$
\exp \left(v_{0} \xi\right)-1=\left(v_{0} / 2 S\right) / f(\zeta) f^{\prime}(\zeta) .
$$


We now consider different cases as $v_{0}>0, v_{0}<0$; and $S>0, S<0$, for an initially sinusoidal pulse given by

$$
f(\zeta)=\sin p \zeta,(p>0)
$$

parallel to the discussions made by Chakraborty (1994).

For $v_{0}>0$ and $S>0$, we have the least possible value of $\xi$ for shock formation as $\left(1 / v_{0}\right) \log \left(1+\left(v_{0} / S p\right)\right)$ for which the concerned values of $\theta$ are given by

$$
\theta=(\pi-2) / 4 p \text { and } \theta=-(3 \pi+2) / 4 p .
$$

Again, for $v_{0}>\theta, S<0$ shock waves are seen to be formed when

$$
\xi=\frac{1}{v_{0}} \log \left(1+\left|\frac{v_{0}}{S_{p}}\right|\right),
$$

for the corresponding value of $\theta$ as

$$
\theta=-((\pi-2) / 2) \text { and } \theta=((3 \pi+2) / 4 p) .
$$

From the above it is seen that the least value of $\xi$ depends upon $v_{0}$, the nonhomogeneity parameter. Hence, the nonhomogeneous character of the material medium has an important role to play in the formation of shocks. Again, it is also observed that the effect of the magnet is to increase the value of $S$ so that least value of $\xi$ decreases as the modulus of $S$ increases. Thus, we see that the joint effect of magnet and the nonhomogeneous character of the material medium cannot be neglected in any way. In the case when $v_{0}<0$ and either $S>0$ or $S<0$, the least positive value of $\xi$ for formation of shocks is

$$
\frac{1}{v_{0}} \log \left(1-\left|\frac{v_{0}}{S p}\right|\right) \text {, where }\left|\frac{v_{0}}{S p}\right|<1 .
$$

Thus, the above study reveals that the formation of shocks is always possible when there is a decay in density, elastic moduli and magnetic permeability, whereas shock formation in the case of growth is possible only when the elastic parameter, the wave frequency and the magnetic field satisfy the condition $\left|v_{0} / S p\right|<1$. The above results are similar in form with those obtained by Chakraborty (1994) for the propagation of quasi-transverse waves in a nonhomogeneous medium but the difference lies in the expression for $S$ which contains the Alfvén wave velocity signifying the effect of the presence of the magnetic field.

\section{Numerical calculations and discussions}

To highlight the effect of magnetic field in the case of a nonlinear quasi-transverse wave propagation in a nonhomogeneous elastic solid medium we plot different types of figures (1 through 6) using numerical calculations with the help of (65) and (66). Comparisons among different cases may also be made with the help of these figures. The figures are self explanatory and exhibit different types of peculiarities of the effects of a magnetic field and nonhomogeneity on the quasi-transverse wave propagation in a nonlinear elastic medium. However, for better understanding of the figures, we present the following explanations. Considering an initially sinusoidal wave given by $f(\theta)=\sin (\pi \theta / 10), h(\theta, \xi)$ has been 

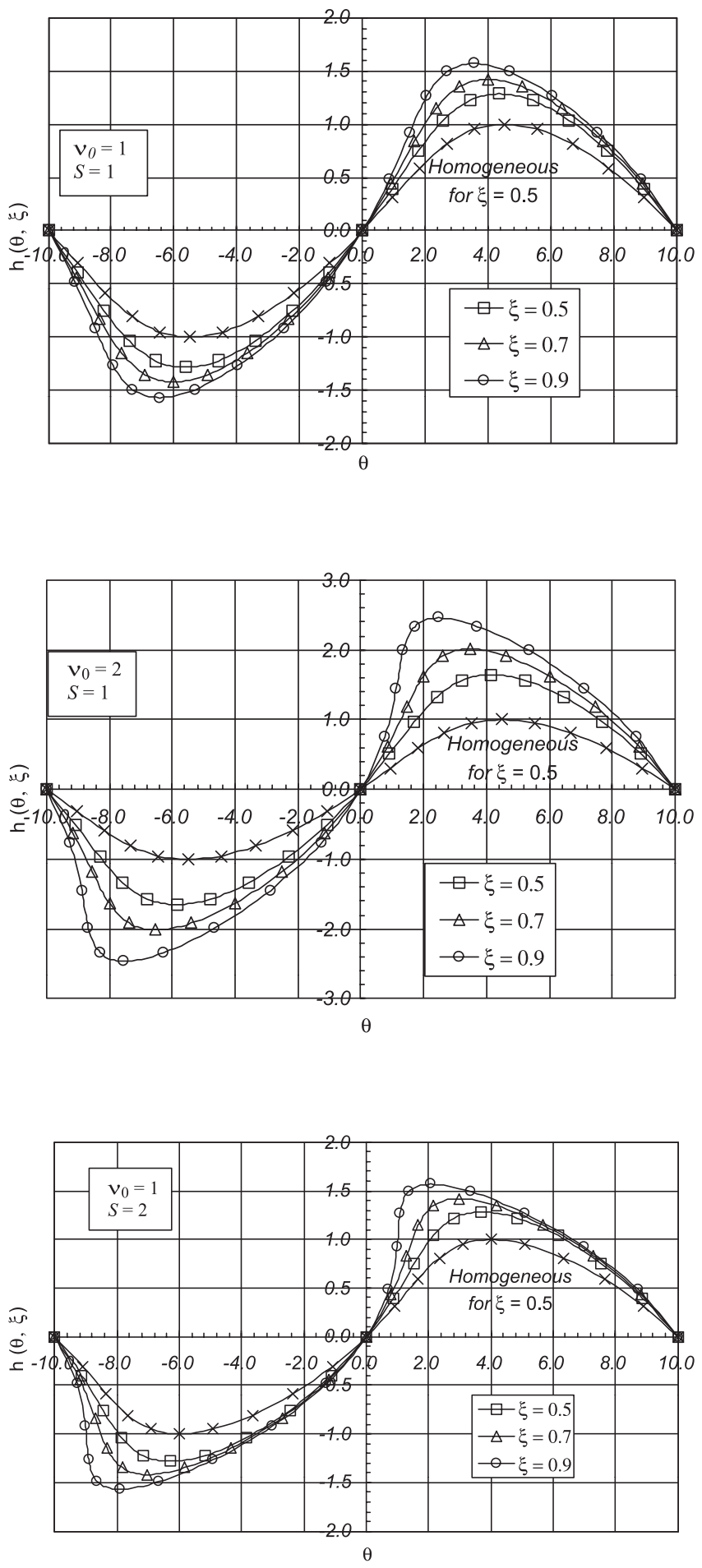

Figure 1. Modulation of $h(\theta, \xi)$ with $\theta$ for a nonhomogenenous nonlinear $\left(v_{0}=1, S=1\right)$ elastic medium for different values of $\xi$.

Figure 2. Modulation of $h(\theta, \xi)$ with $\theta$ for a nonhomogenenous nonlinear $\left(v_{0}=2, S=1\right)$ elastic medium for different values of $\xi$.

Figure 3. Modulation of $h(\theta, \xi)$ with $\theta$ for a nonhomogenenous nonlinear magnetoelastic $\left(v_{0}=\right.$ $1, S=2$ ) medium for different values of $\xi$. 

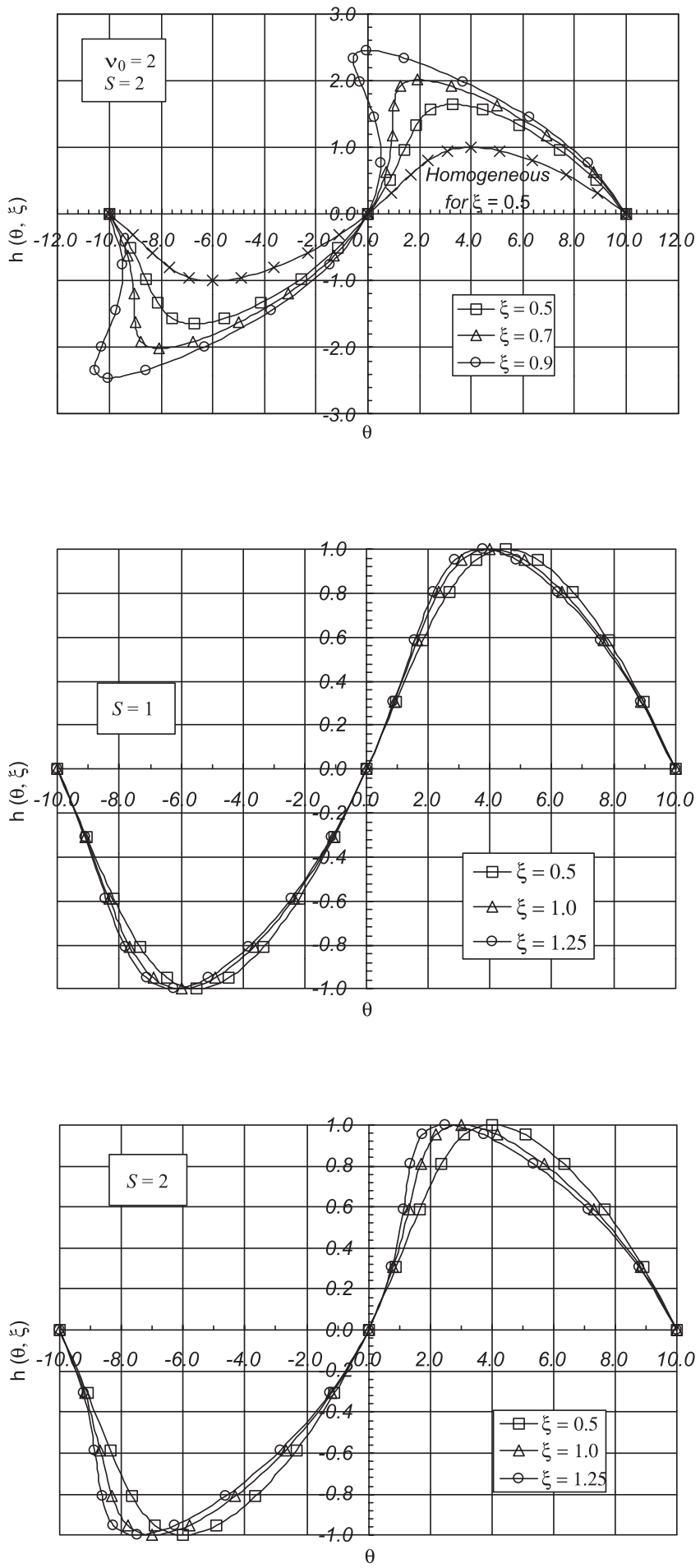

Figure 4. Modulation of $h(\theta, \xi)$ with $\theta$ for a nonhomogenenous nonlinear magnetoelastic $\left(v_{0}=\right.$ $2, S=2$ ) medium for different values of $\xi$.

Figure 5. Modulation of $h(\theta, \xi)$ with $\theta$ for a nonhomogenenous nonlinear $\left(v_{0}=0, S=1\right)$ elastic medium for different values of $\xi$.

Figure 6. Modulation of $h(\theta, \xi)$ with $\theta$ for a nonhomogenenous nonlinear $\left(v_{0}=0, S=2\right)$ elastic medium for different values of $\xi$. 
plotted against $\theta$ for different values of $\xi$. Figures $1\left(v_{0}=1, S=1\right)$ and $2\left(v_{0}=2, S=1\right)$ correspond to the propagation of quasi-transverse waves in a nonhomogeneous nonlinear elastic medium for different values of $\xi$. For comparison we have also drawn the corresponding curves for a homogeneous medium with $\xi=0.5$ only. It is also observed that $h(\theta, \xi)$ increases with increase of $\xi$. The peculiarities of different curves are noticeable. The least value of $\xi$ for which shocks are formed are 1.43 for $v_{0}=1$ and 0.9985 for $v_{0}=2$ respectively. Figures 3 $\left(v_{0}=1, S=2\right)$ and $4\left(v_{0}=2, S=2\right)$ express the modulation of the values of $h(\theta, \xi)$ in the case of wave propagation in a nonhomogeneous nonlinear magneto-elastic medium. In this case, the least value of $\xi$ for which shocks are formed have been calculated as $\xi=0.95$ for $v_{0}=1$ and $\xi=0.7155$ for $v_{0}=2$ respectively. Curves of figure 5 have been drawn for the propagation of quasi-transverse waves in a homogeneous nonlinear elastic medium for different values of $\xi$, whereas those of figure 6 correspond to the propagation of quasitransverse waves in a nonlinear magneto-elastic homogeneous medium. In each of the figures, distortion of wave form occurs as the wave progresses. Graphs show that the distortion is not symmetric for positive and negative parts of the waves which is in conformity with the previous analysis in the bulk of the paper. In figures 1, 2, $3 \& 4$ the symbol $v_{0}$ has been used for the previously used symbol $v_{0}$.

\section{Conclusion}

The most significant outcome of the paper is that the magnetic field as well as nonhomogeneous character of the material medium modulates the quasi-transverse wave propagation in a nonlinear elastic medium to a considerable extent. Further modulation occurs due to their joint effect.

Thanks are due to Mr. Sourav Acharya, Indian Institute of Technology, Madras, Chennai for his computational help with the numerical calculations and graphs in this paper.

The authors take this opportunity to express their gratitude to the reviewers for their valuable comments and suggestions towards the improvement of this paper.

\section{References}

Ang W T, Clements D L 1987 On some crack problems for inhomogeneous elastic materials. Int. J. Solids Struct. 23: 1089-1104

Bazer J 1984 A survey of linear and nonlinear wave motion in a perfect magneto-elastic medium. In The mechanical behaviour of electro magneto solid continua (ed.) G A Maugin (Amsterdam: Elsevier Science-North Holland)

Bland D R 1969 Nonlinear dynamic elasticity (Waltham, MA: Blaisdell)

Chakraborty S 1994 Effect of nonhomogeneity on quasi-transverse waves in a nonlinear elastic medium. Indian J. Pure Appl. Math. 24: 467-474

Chakraborty S 1998 On distortion of waves in a nonlinear magneto elastic conductor. Arch. Mech. 50: 1047-1053

Chandrasekharaiah D S 1987a Effects of surface stresses and voids on Rayleigh waves in an elastic solid. Int. J. Eng. Sci. 25: 205-211

Chandrasekharaiah D S 1987b Plane waves in a rotating elastic solid with voids. Int. J. Eng. Sci. 25: $591-596$ 
Chattopadhyay A, Pal A K, Chakraborty M 1984 SH waves due to a point source in an inhomogeneous medium. Int. J. Nonlinear Mech. 19: 53-60

Eringen A C, Suhubi E S 1974 Elastodynamics, vol. 1: Finite motions (New York and London: Academic Press)

Hefni I A Z, Ghaleb A A, Maugin G A 1995 Surface waves in a nonlinear magneto-elastic conductor of finite electric conductivity. Int. J. Eng. Sci. 33: 2085-2102

Lardner R W 1983 Nonlinear surface waves in an elastic solid, Int. J. Eng. Sci. 21: 1331-1342

Lardner R W 1985 Nonlinear effects on transverse shear waves in an elastic medium. J. Elasticity 15: 53-57

Maugin G A 1981 Wave motion in magnetizable deformable solids. Int. J. Eng. Sci. 19: 321-388

Nayfeh A H 1973 Perturbation methods (New York: John Wiley and Sons)

Neyfeh A H 1975 Finite longitudinal waves in nonuniform bar. J. Sound Vibr. 42: 353-361

Pal P K, Acharya D 1998 Effects of inhomogeneity on surface waves in anisotropic media. Sadhana 23: 247-258

Paria G 1967 Magneto elasticity and magneto-thermo elasticity. Adv. Appl. Mech. 10: 73-112

Semra A, Mevlut T 2004 Rayleigh-like surface waves on a nonlinear layered elastic half space. Twentyfirst Int. Conf. of Theoretical \& Applied Mechanics (XXI-ICTAM) Warsaw, Poland, pp 15-21

Truesdell C, Noll W 1965 The nonlinear field theories of mechanics, theories of mechanics. In Encyclopedia of physics (ed.) S Flugge (Berlin: Springer-Verlag) vol. 111(3)

Yu C, Tang S 1966 Magneto-elastic waves in initially stressed conductors. Z. Angew. Math. Phys. 17: 766-775 